RELACIÓN ENTRE IMPULSIVIDAD, AFRONTAMIENTO Y TOMA DE DECISIONES DE CONTINUAR O NO LA RELACIÓN CON EL AGRESOR EN MUJERES QUE DENUNCIARON VIOLENCIA DE PAREJA

ESPERANZA PALACIOS CORTÉS

Directora de Tesis:

ÁNGELA CRISTINA TAPIAS SALDAÑA

UNIVERSIDAD SANTO TOMÁS

MAESTRÍA EN PSICOLOGÍA JURÍDICA

BOGOTÁ, DC, JUNIO DE 2016 


\section{Relación entre impulsividad, afrontamiento y toma de decisiones de continuar o no la relación con el agresor en mujeres que denunciaron violencia de pareja}

\section{$\underline{\text { Resumen }}$}

La violencia contra la mujer es una violación a los derechos humanos, la presente investigación profundiza en el entendimiento psicojurídico de este fenómeno, aplica un enfoque descriptivo y correlacional utilizando las variables de impulsividad, afrontamiento y toma de decisión de continuar o no la relación con el agresor, en 42 casos de mujeres que solicitaron medida de protección ante la Comisaría de Familia de Chapinero. Los resultados del estudio muestran una correlación positiva entre las mujeres que no conviven con el agresor y las que presentan mayor autonomía como estilo de afrontamiento, así como entre impulsividad y reacción agresiva, e impulsividad con dificultad de afrontamiento, lo cual explica que se mantenga la problemática. Sin embargo, contrario a lo esperado se encontró que a mayor impulsividad hubo mayor espera, lo que puede asociarse a los ciclos de violencia con períodos de tolerancia y descontrol. Como aportes de este trabajo, se ofrecen los hallazgos encontrados para un mejor abordaje de la violencia de pareja desde la psicología y el derecho.

Palabras clave: Impulsividad, estrategias de afrontamiento y violencia de pareja. 


\section{Relationship between impulsivity, coping and decision-making on whether to continue the relation with the aggressor in the case of women who reported intimate partner violence}

\section{$\underline{\text { Abstract }}$}

Violence against women in the context of couple relations is a clear violation of human rights. With the aim of expanding the understanding of this phenomenon, this paper applies a descriptive and correlative approach using the variables of impulsivity, coping and decisionmaking on whether to continue the relationship with the aggressor in the case of 42 women who requested protection measures at Comisaría de Familia de Chapinero. The results show a positive correlation between women who do not live with the aggressor and those who present more autonomy as a way of coping; between impulsiveness and aggressive reactions; and between impulsivity and difficulty for coping, which could explain the persistence of the problem. However, contrary to the initial expectation the study found that greater impulsivity correlates with longer times to react, which may be associated with cycles of violence characterized by periods of tolerance and lack of control. As contributions of this work, findings are offered for better addressing intimate partner violence under the light of psychology and laws.

Keywords: impulsivity; coping strategies; partner violence

La violencia contra la mujer es una clara violación a los derechos humanos (Sánchez, Encisco, Arenas, Vergara y Lanz, 2012), es un problema social y de salud pública (OMS, 2002); una mujer víctima de violencia necesita un promedio de diez años para reconocer que está siendo maltratada, denunciar este maltrato y acabar con su matrimonio (Vásquez, 2007). Cantón, Cortés, Justicia y Cantón (2013) plantean, como fundamental la investigación sobre la etiología, mantenimiento y consecuencias de la violencia de pareja, por lo que, dentro de este contexto, radica la importancia de comprender cómo se relacionan las estrategias de afrontamiento y la 
impulsividad con la toma de decisión de separarse o no de su pareja en mujeres víctimas de maltrato y ahondar en el entendimiento integral de la violencia de pareja.

De esta manera, el presente estudio, pretende profundizar en la comprensión de las relaciones humanas y la reacción social vinculada a la decisión legal (medida de protección), conocer los factores que mantienen o extinguen la relación violenta, a través de tomar decisiones que rescaten la dignidad de las mujeres y que permitan generar mejores estrategias de detección, atención y de prevención, además optimizar el abordaje psicojurídico, campo en donde la conducta tiene relación y consecuencia legales. Así mismo, para entender esta problemática se realiza un recorrido teórico por tres aspectos básicos: violencia de pareja desde la perspectiva de la psicología jurídica, impulsividad, así como de estrategias de afrontamiento.

La violencia de pareja, para Rolling y Brosi (2010), consiste en el uso de una pauta repetida de comportamientos coercitivos y controladores para limitar, dirigir y disponer los pensamientos, sentimientos y acciones de la pareja sentimental; mientras que para Taggart (2009) es un patrón de comportamiento en el que una persona intenta controlar a su pareja a través de amenazas, violencia física, asalto sexual, abuso verbal y psicológico y coerción económica. Para ilustrar mejor, Pueyo, López y Álvarez (2008) la definen como el conjunto complejo de distintos tipos de comportamientos violentos, actitudes, sentimientos, prácticas, vivencias y estilos de relación entre miembros de una pareja (o ex pareja) íntima que produce daños, malestar y perdidas personales graves a la víctima.

Para comprender el fenómeno de la violencia de pareja, Corsi (2004) plantea la necesidad de analizar los factores que la legitiman culturalmente, las creencias y los valores acerca de las mujeres y de los hombres en una sociedad patriarcal que define a los varones como superiores por naturaleza y les confiere el derecho y la responsabilidad de dirigir la conducta de su mujer. 
Estas actitudes y valores, se traducen en estructuras sociales particulares como la división del trabajo, las políticas institucionales y la discriminación de la mujer.

De acuerdo con Walker (1989), citado en Labrador, Rincón, Luis y Fernández-Velasco (2004) la "Teoría del ciclo de la violencia" plantea tres fases. Primera: acumulación de tensión; segunda: descarga de la violencia y tercera: arrepentimiento. En este ciclo se dan tres características fundamentales, según Labrador, Rincón, Luis y Fernández-Velasco (2004): primero: cuantas más veces se completa el ciclo, menos tiempo necesita para completarse; segundo: la intensidad y la severidad de la violencia va aumentando progresivamente con el tiempo, llegando incluso a la muerte; tercero: la fase del arrepentimiento tiende a hacerse más corta y puede desaparecer con el tiempo, se va creando un hábito en el uso de la violencia, este ciclo tiende a no detenerse por sí mismo.

La problemática de la violencia de pareja, constituye un problema de salud pública presente en varios países; según Breiding, Basile, Smith, Black y Mahendra (2015) afecta a millones de estadounidenses y contemplan la violencia no sólo física, sino también la sexual, el acoso y la agresión psicológica, ejercida por la pareja actual o anterior, se presenta como un proceso continuo, que va desde un episodio a episodios crónicos y severos durante varios años. Por su parte, Bell, y Naugle, (2008) aportan una explicación holística y plantean distintas etiologías de este fenómeno, distinguiendo diferentes agentes causantes y describiendo su forma de interactuar, tales como factores socioculturales, características individuales tanto del agresor como de la víctima, tipologías de agresores, y modelos funcionales.

A su vez, para la Secretaría Distrital de Integración Social (2015) la violencia de pareja es un problema nodal que afecta el tejido social, ocurre en el entorno familiar, lugar protector que se transforma en un espacio de riesgo para los integrantes de la familia; los sistemas de información 
no dan cuenta real de las estadísticas ya que no se visibiliza y permanece oculta. Sin embargo, los resultados de la Encuesta Nacional de Mujeres Contra la Violencia (USA), demuestran que las víctimas son atacadas cada vez con más frecuencia y es menos probable que la violencia se detenga; son más propensas a sufrir lesiones, estrés postraumático, al uso de analgésicos y a faltar al trabajo (Johnson, 2005).

Las diferencias psicológicas de género, la cultura patriarcal y la tradición de mitos, generan en la mujer la responsabilidad de mantener la familia, favorecen el establecimiento y mantenimiento de violencia y ayudan a que podamos comprender por qué una mujer maltratada normaliza la violencia (Vásquez, 2007). Entre otros motivos, según la Encuesta Nacional de Demografía y Salud (Profamilia, 2010), se encuentran la creencia de las mujeres que pueden resolver el problema solas (24\%), las secuelas no fueron muy graves (22\%), miedo a un mayor maltrato (16\%), no desea afectar al agresor (13\%), no cree que la escena violenta vuelva a ocurrir (9\%), es parte de la vida normal (9\%), ignora las entidades a dónde ir a oponer la denuncia (7\%)

Según el Instituto Nacional de Medicina Legal y Ciencias Forenses (2013), la mayoría de los casos se presentan entre los 25 a 29 años de edad; entre los principales presuntos agresores se encontró al compañero permanente, seguido del ex compañero y luego, el esposo. La violencia contra la pareja durante el año 2014 tuvo un incremento de 4.106 valoraciones médico legales en comparación con el año 2013, es decir, una tasa de 125 por cada 100.000 habitantes, la cual supera la del año 2013, que fue de 95 por cada 100.000 habitantes (Instituto Nacional de Medicina Legal y Ciencias Forenses, 2014). Así mismo, en el año 2015 en el Instituto en mención se registraron 47.248 casos de violencia de pareja.

No obstante, que la normatividad en Colombia es amplia y reconoce los derechos de las mujeres, se encuentran falencias para el logro efectivo de éstos derechos (Sánchez, Encisco, 
Arenas, Vergara y Lanz, 2012). La caracterización descriptiva de la violencia de pareja aporta elementos importantes para la contextualización, valoración del riesgo y brinda a las autoridades competentes las herramientas para la adopción de medidas de protección y atención de las víctimas de conformidad con la Ley por el derecho de las mujeres a una vida libre de violencia (Instituto Nacional de Medicina Legal y Ciencias Forenses, 2014). Esta ley da un enfoque integral al problema de violencia contra las mujeres, abarca el daño psicológico, físico, sexual, patrimonial, en el ámbito público como privado, incluye al sector salud, justicia, sociedad en general, y establece la posibilidad de adoptar Medidas de Protección de obligatoria competencia de las Comisarias de Familia (Ministerio de Justicia y del Derecho, 2012).

Con base en esto, las Comisarías de Familia tienen competencia para recibir denuncias de violencia de pareja y realizar acciones de prevención de nuevos hechos. Sin embargo, las consultas que reciben crecen exponencialmente y desbordan la capacidad de respuesta institucional (Secretaría Distrital de Integración Social, 2015, p.8). En este contexto, se hace prioritario el conocimiento de la legislación sobre violencias, como herramienta para ejercer los derechos, el conocimiento de las rutas de atención, el empoderamiento de las mujeres víctimas, la deconstrucción de imaginarios culturales que naturalizan y validan la violencia como estrategias para hacer frente y prevenir este fenómeno que genera dolor e innumerables consecuencias (Secretaría Distrital de Integración Social, 2015).

De manera paralela, se realiza la revisión de definiciones de la variable de estudio: impulsividad, concepto complejo y de difícil reducción a un constructo unitario; incluso, existen diferencias entre autores al tratar de definirlo, comprende la planeación, la toma de decisiones, la autorregulación emocional y la motivación; se relaciona con bases biológicas, cambios en el desarrollo psicológico y adquisición de habilidades y de herramientas (Sánchez, Giraldo y 
Quiroz, 2013; Squillace, Picón y Schmidt, 2011). Es definida como un rasgo de personalidad dimensional, caracterizado por el déficit en la inhibición de la conducta, el cambio de comportamiento ante la demanda del medio ambiente y la tolerancia para el retraso de la gratificación (Reynolds y Schiffbauer, 2005; Swann, Bjork, Moeller y Dougherty, 2002).

La impulsividad, del latín impulsus (golpear o empujar), de acuerdo con Orozco- Cabal y Herin (2008) y Haro, Castellano, Pérez, Rodríguez, Cervera1 y Valderrama (2004), es definida como la falta de control en el comportamiento y ejecución de acciones que minimizan el tiempo para recibir una recompensa; en concordancia con esto, Sánchez, Giraldo y Quiroz (2013), plantean que, al definir la impulsividad, se debe hacer referencia a tres factores: el actuar sin una implicación directa de las funciones del lóbulo frontal, un aumento en la velocidad de la respuesta emitida y una obtención inmediata de gratificación. Por su parte, diferentes corrientes psicológicas, hacen hincapié en dos aspectos puntuales de la impulsividad: capacidad de autocontrol para el retraso de la gratificación y proceso de toma de decisiones (Kidd, Palmeri y Aslin, 2013; Casey, Somerville, Gotlib, Ayduk, Franklin, Askren y Shoda, 2011; Martínez, Sánchez, Bechara y Román, 2006).

A pesar de que ha sido un concepto difícil de definir, a través de los hallazgos en sus estudios, varios autores coincidieron en algunos aspectos en común, que han de ser tenidos en consideración. En primer lugar, comportamientos dirigidos hacia la búsqueda de actividades, refuerzos positivos y a la evitación de frustración; adicional a esto, encontraron conductas de evitación sostenida o de comportamientos compulsivos para atenuar la tensión, producto del estrés; coincidieron, además, en que los individuos impulsivos exhiben la ejecución de actos rápidos y no premeditados, debido a la falta de conciencia o a la minimización de las consecuencias; y finalmente, concordaron en que la impulsividad, en altos niveles, se suele 
relacionar con trastornos o desordenes psicológicos (Squillace, Picón y Schmidt, 2011; Smith, Fischer, Cyders, Annus, Spillane y McCarthy, 2007; Winstanley, Eagle y Robbins, 2006; Chahin, Cosi, Lorenzo-Seva y Vigil-Colet, 2005).

Cabe señalar, también, lo planteado por Portilla, Bascarán, Sáiz, Bousoño y Bobes (2005), que definen la impulsividad como una dificultad comportamental y cognitiva, es una reacción inmediata que no tiene en cuenta las consecuencias y que se ha identificado en el 10-15\% de la población general, presentando un significativo desgaste en el sector salud y asistencial, así como el sufrimiento personal, las dificultades psicosociales y laborales.

Continuando con la investigación, se realiza la revisión teórica de la variable: estrategias de afrontamiento, definidas como un conjunto de recursos y esfuerzos tanto cognitivos como comportamentales orientados a enfrentar situaciones estresantes (Lazarus y Folkman, 1986). Pueden focalizarse en el problema, en la emoción o en la valoración de la situación (FernándezAbascal y Palmero, 1999). Lazarus y Folkman (1984) explican el afrontamiento como respuesta para manejar las demandas específicas externas e internas apreciadas como excedentes o que desbordan los recursos del individuo (p. 141), tiene un componente altamente subjetivo en el que el sujeto hace "lo que puede, con lo que tiene".

En este orden de ideas, Morales (2008) define la estrategia como "el conjunto de acciones que se ponen en juego para solucionar o satisfacer unas demandas específicas del estresor" (p. 47), dependen de las características del contexto, pueden ser "adaptativas o no". Frente a un mismo acontecimiento estresante cada individuo presenta una reacción muy diversa, gira en torno a las estrategias de afrontamiento o coping, y busca determinar, cómo se enfrenta cada individuo a situaciones estresantes, sean graves o problemas de la cotidianidad (Vásquez, Crespo y Ring, 2003; Stanton y Dunkel, 2013). 
De manera específica, el afrontamiento se refiere a las habilidades individuales y culturales que involucran una serie de pensamientos y acciones, orientadas hacia el manejo de conflictos (Ojeda, Cuenca y Espinosa, 2008), comprende una serie de esfuerzos dirigidos a manejar, lo mejor posible, las demandas internas y ambientales. Se dividen en dos grandes grupos: estrategias con una tendencia evitadora o minimizadora de la situación, mientras que las otras, son confrontadoras, sea buscando información o ejecutando acción directa (Vásquez, Crespo y Ring, 2003; Flórez, Herrera, Carpio, Veccino, Zambrano, Reyes y Torres, 2011).

Las estrategias de afrontamiento hacen visibles las características personales, los pensamientos, los sentimientos y las acciones individuales propias de un ser holístico quien, al utilizar los recursos de afrontamiento innatos y adquiridos, busca adaptarse a un ambiente cambiante (Veloza, Moreno, Crespo y Gutiérrez, 2007). Aunque la puesta en marcha de las estrategias de afrontamiento no siempre garantiza el éxito, sirven para generar, evitar o disminuir conflictos en los seres humanos, atribuyéndoles beneficios personales y contribuyendo a su fortalecimiento (Zambrano, Valle, Macías y Madariaga, 2013).

Se ha evidenciado cómo las redes de apoyo social: familia, amigos, compañeros de trabajo son indispensables para una mujer atrapada en un ciclo de violencia y cómo los medios materiales son fundamentales para el proceso de toma de decisiones, así como el apoyo emocional, los procedimientos legales, la comunidad, servicios terapéuticos, las creencias religiosas, las instituciones, la espiritualidad y los recursos internos (el locus de control, autovaloración, autoestima). Aunque existen muchas tácticas potenciales, las mujeres las utilizan en diferentes formas dependiendo de las estrategias preferidas de afrontamiento, la percepción de utilidad y el tipo de comunidad (Goodman, Dutton, Vankos y Weinfurt, 2005). 
Por su parte, Echeburúa, Corral y Amor (2002) plantean cómo un apoyo social próximo insuficiente, la depresión, el aislamiento y escasas relaciones sociales, dificultan la recuperación de las víctimas. Por lo tanto, los autores proponen como estrategias de afrontamiento: la aceptación del hecho, compartir la experiencia, reinterpretación positiva del suceso, establecimiento de nuevas metas y relaciones, búsqueda de apoyo social, vinculación en grupos de autoayuda o en ONG y la búsqueda de apoyo social institucional, como el sistema judicial, la policía para que las mujeres víctimas de violencia de pareja tengan mayor resistencia al estrés, sean capaces de hacer frente a las experiencias maltratadoras y tomar mejores decisiones.

Al llegar a este punto, dentro del marco teórico analizado, es importante revisar algunas investigaciones: se han encontrado estudios de abuso emocional en relación con el abuso físico. Se ha observado como el ridículo, las humillaciones y el control excesivo pueden ser más perjudicial para la salud mental que algunos actos de violencia física (Copp, Giordano, Longmore y Manning, 2015; Rhatigan, Street y Axsom, 2006 y Campbell, 2002). Los resultados hallados por Shannon, Logan, Cole y Medley (2006), evidencian que las mujeres urbanas utilizan más recursos de búsqueda de ayuda que las mujeres rurales, las mujeres rurales perciben los servicios del sistema de justicia como menos útiles que las mujeres urbanas.

La mayoría de los estudios sobre afrontamiento (Perona y Galán, 2001; Mestre, Samper, Tur-Porcar, Richaud de Minzi y Mesurado,2012), coinciden en determinar tres dominios según estén dirigidas a la valoración (afrontamiento cognitivo: intento de encontrar significado al suceso y valorarlo de manera que resulte menos desagradable), dirigidas al problema (afrontamiento conductual: conducta dirigida a confrontar la realidad, manejando sus consecuencias) y a la emoción (afrontamiento afectivo: regulación de los aspectos emocionales e intento de mantener el equilibrio afectivo). 
Por su parte, Murray y Ali (2016) encuentran que el afrontamiento activo, la planificación para hacer frente a situaciones de estrés y la toma de decisiones es importante para las minorías étnicas y que la adaptación basada en la emoción, se utiliza cuando la planificación activa es menos frecuente. Mientras que De la Rubia y López (2011), plantean cómo el afrontamiento pasivo es crítico para recibir violencia. El evitar los conflictos, acomodarse sin luchar y desestimar la negociación y búsqueda conjunta de soluciones es el motor de la violencia, ya que incrementa los problemas entre los miembros de la pareja y la irritación en el otro.

Así mismo, Engin (2006) evidenció que la satisfacción con la vida se correlaciona positivamente con el afrontamiento centrado en el problema y la búsqueda de apoyo social y cómo la satisfacción de vida se correlaciona significativamente con todos los estilos de toma de decisiones. Además, encontró relaciones significativas entre afrontamiento del estrés y estilos de toma de decisiones. Los distintos trabajos realizados por Martínez, Piqueras y Ramos (2010) muestran que niveles altos en inteligencia emocional se relacionan con estrategias de afrontamiento basadas en la reflexión y la resolución de problemas, mientras niveles bajos se relacionan con estrategias de afrontamiento basadas en la evitación, la rumiación y la superstición

En el estudio de Averdijk, Van Gelder, Eisner y Ribeaud (2016), se parte de la hipótesis que haber sido víctima de violencia, afecta a la valoración de un individuo de situaciones potencialmente conflictivas posteriores, las víctimas se vuelven más sensibles hacia los beneficios de la perpetración de violencia que hacia sus costos. Los resultados del análisis, mostraron que la trayectoria de la victimización previa influyó en la apreciación de situaciones de toma de decisiones. Rhatigan et al. (2006) plantean como la gente suele preguntar: "¿Por qué algunas personas permanecen en relaciones violentas?" Este estudio encuentra que la tasa de la 
terminación de la relación entre el subgrupo IPV (violencia del compañero íntimo), fue comparable a las tasas del subgrupo no violento; la literatura previa ha sugerido que la permanencia de parejas con IPV es similar a la de parejas no violentas.

Por su parte, la investigación realizada por Sánchez (2015), determinó que la impulsividad, es decir pensar y actuar de manera inmediata y sin medir las consecuencias de los actos en los diferentes aspectos de la vida cotidiana, se relaciona directamente con el riesgo de violencia contra la pareja, significando que, las personas que presentaron impulsividad, ya sea cognitiva, motora o improvisación y ausencia de planificación guardan una estrecha relación con el riesgo de violencia.

Teniendo en cuenta la revisión teórica realizada, se plantea como pregunta de investigación: ¿Cuáles son las relaciones entre impulsividad, afrontamiento y la decisión de continuar o no la relación con el agresor en mujeres que denunciaron violencia de pareja y se les otorgó Medida de Protección? Por tanto, el objetivo del presente estudio fue describir y correlacionar las variables impulsividad, afrontamiento y toma de decisión de continuar o no la relación con el agresor en mujeres que denunciaron violencia de pareja. Se generaron las siguientes hipótesis: ¿Las mujeres víctimas de violencia de pareja que presentan mayor impulsividad siguen conviviendo con su agresor?, a mayor impulsividad menor espera como estrategia de afrontamiento?; a mayor autonomía se separa de su agresor?; a mayor búsqueda de apoyo profesional continúa con su pareja?

\section{Método}

\section{Participantes}


La muestra está compuesta por cuarenta y dos (42) mujeres, a quienes se les otorgó medida de protección por violencia de pareja ante la Comisaría de Familia de Chapinero durante los años 2014 al 2016, divididas en dos (2) grupos: 21 mujeres que continúan viviendo con su agresor y 21 mujeres que se separaron de ellos. La edad media fue de 35,2 años, con rangos que oscilaron entre los 19 y 63 años, las edades más frecuentes fueron 28 y 38 años. El nivel educativo fluctuó entre primaria incompleta a especialización, siendo representativo que el 29\% cursó bachillerato incompleto y el $24 \%$ bachillerato completo. El estrato socioeconómico se reportó del 1 al 5 , siendo el $55 \%$ de las mujeres estrato 2.

Tabla 1.

Datos sociodemográficos de las participantes.

\begin{tabular}{|c|c|c|c|c|}
\hline PARTICIPANTE & EDAD & ESTRATO & ESTUDIOS & OCUPACION \\
\hline 1 & 38 & 2 & Bachiller Incompleto & Empleada \\
\hline 2 & 31 & 2 & Técnico & Hogar \\
\hline 3 & 23 & 2 & Técnico & Empleada \\
\hline 4 & 56 & 4 & Bachiller & Independiente \\
\hline 5 & 38 & 2 & Profesional & Contratista \\
\hline 6 & 21 & 1 & Bachiller & Empleada \\
\hline 7 & 38 & 5 & Universidad Incompleta & Independiente \\
\hline 8 & 20 & 1 & Bachiller Incompleto & Hogar \\
\hline 9 & 34 & 2 & Primaria & Hogar \\
\hline 10 & 63 & 2 & Primaria Incompleta & Hogar \\
\hline 11 & 21 & 1 & Primaria & Hogar \\
\hline 12 & 48 & 4 & Bachiller & Independiente \\
\hline 13 & 24 & 1 & Bachiller Incompleto & Independiente \\
\hline 14 & 50 & 3 & Universidad Incompleta & Empleada \\
\hline 15 & 28 & 2 & Bachiller & Empleada \\
\hline 16 & 35 & 2 & Bachiller & Empleada \\
\hline 17 & 28 & 1 & Universidad Incompleta & Empleada \\
\hline 18 & 20 & 2 & Técnico & Empleada \\
\hline 19 & 52 & 4 & Técnico & Hogar \\
\hline 20 & 32 & 4 & Universitaria & Empleada \\
\hline 21 & 28 & 2 & Primaria & Independiente \\
\hline 22 & 26 & 2 & Bachiller Incompleto & Hogar \\
\hline 23 & 56 & 2 & Bachiller Incompleto & Independiente \\
\hline 24 & 57 & 2 & Primaria & Hogar \\
\hline 25 & 33 & 4 & Universidad Incompleta & Independiente \\
\hline 26 & 55 & 2 & Primaria & Empleada \\
\hline 27 & 29 & 2 & Bachiller & Empleada \\
\hline 28 & 29 & 2 & Bachiller Incompleto & Empleada \\
\hline 29 & 38 & 4 & Bachiller & Empleada \\
\hline
\end{tabular}




\begin{tabular}{|c|c|c|c|}
\hline 30 & 27 & 2 Técnico & Hogar \\
\hline 31 & 21 & 2 Bachiller & Empleada \\
\hline 32 & 23 & 2 Bachiller & Hogar \\
\hline 33 & 38 & 2 Bachiller Incompleto & Empleada \\
\hline 34 & 28 & 1 Bachiller & Hogar \\
\hline 35 & 35 & 2 Bachiller Incompleto & Empleada \\
\hline 36 & 20 & 2 Bachiller Incompleto & Empleada \\
\hline 37 & 28 & 1 Bachiller Incompleto & Hogar \\
\hline 38 & 55 & 4 Especialización & Empleada \\
\hline 39 & 50 & 3 Primaria & Hogar \\
\hline 40 & 19 & 3 Primaria & Empleada \\
\hline 41 & 39 & 2 Bachiller Incompleto & Hogar \\
\hline 42 & 37 & 5 Bachiller Incompleto & Empleada \\
\hline
\end{tabular}

\section{Tipo y diseño de investigación}

El paradigma de conocimiento es el positivismo, el enfoque de la investigación es cuantitativo; el diseño de tipo descriptivo y correlacional, de corte transversal.

\section{Instrumentos}

Encuesta general sobre la Medida de Protección, tipo Likert.

Mide el conocimiento de los derechos fundamentales de las mujeres de la muestra antes de acudir a la Comisaria de Familia, su opinión sobre la Medida de Protección y las orientaciones que recibió por parte de la Comisaria y si éstas orientaciones influyeron en la toma de decisión de separarse o no de su pareja; por otro lado, la aplicación de este instrumento ayudó a conformar los grupos del presente estudio.

Escala de impulsividad de Barratt (BIS).

Evalúa la impulsividad asociada con las decisiones, punto de corte 74. Loyola (2011) analizó la confiabilidad del BIS-11 a partir de la consistencia interna de los reactivos para cada uno de los factores que componen la prueba. Los resultados obtenidos indicaron que el BIS-11 cuenta con una confiabilidad aceptable.

Escala de Estrategias de Coping Modificada (EEC-M)_en una muestra colombiana. 
Valora los recursos orientados a enfrentar situaciones estresantes, se identificaron las propiedades psicométricas y validez estructural; define doce factores: solución de problemas (SP), búsqueda de apoyo social (BAS), espera (ESP), religión (REL), evitación emocional (EE), búsqueda de apoyo profesional (BAP), reacción agresiva (RA), evitación cognitiva (EC), reevaluación positiva (RP), expresión de la dificultad de afrontamiento (EDA), negación (NEG) y autonomía (AUT) (Londoño, Henao, Puerta, Posada, Arango y Aguirre-Acevedo, 2006).

\section{Procedimiento}

Se aplicaron los instrumentos mencionados a las mujeres de la muestra, previo diligenciamiento del Consentimiento Informado, conforme al Literal I, Art. 36, Cap.III, Título VII, Ley 1090 de 2006, que estipula los deberes del psicólogo con las personas en su ejercicio profesional. Paralelamente, se les informó el objetivo de la investigación y las condiciones éticas del mismo, las mujeres manifestaron su participación voluntaria y autorizaron la aplicación de los instrumentos, se indicó que la información derivada será publicada en un informe científico y se realizará retroalimentación individual.

\section{Resultados}

En la encuesta general sobre la medida de protección, el 55\% de las mujeres de la presente investigación reportaron que no conocían sus derechos fundamentales como mujer antes de acudir a la Comisaria de Familia; en lo referente a las orientaciones que recibieron por parte la Comisaria de Familia, el $48 \%$ de las mujeres consideraron que fueron útiles y el $36 \%$ claras; manifestaron que la medida de protección que se le otorgó por parte de la Comisaria de Familia fue oportuna el $52 \%$ y el $31 \%$ efectiva; el $57 \%$ de las mujeres expresó que las orientaciones que recibió por parte la Comisaria de Familia no influyeron en la toma de decisión de separarse o no 
de su pareja; después de la medida de protección el $50 \%$ continuo viviendo con su agresor y el 50\% de las mujeres que se separó de ellos, éste ítem permitió conformar la muestra.

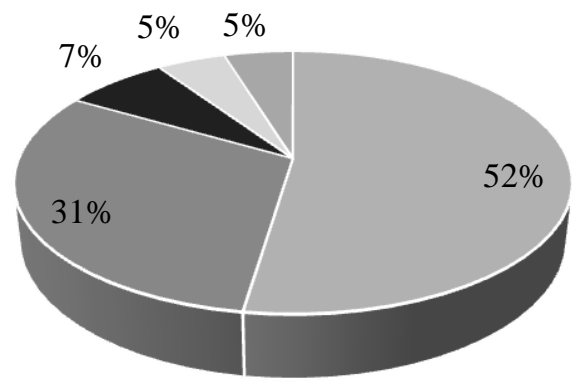

$52 \%$ Oportuna

$31 \%$ Efectiva

$7 \%$ Accesible

$5 \%$ Desproporcionada

$5 \%$ Inútil

$0 \%$ Ineficiente

Figura 1. Resultado de la medida de protección.

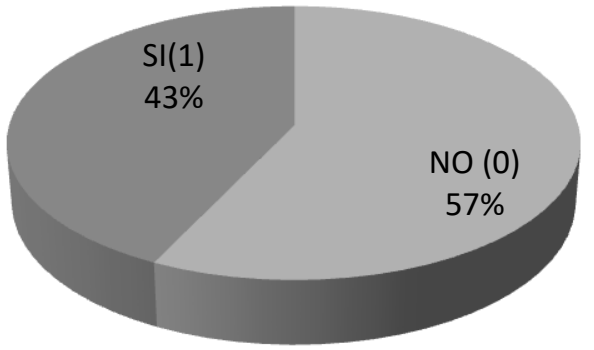

Figura 2. Influencia de la Comisaría sobre la toma de decisión.

Después de elaborar la base de datos, se procedió a realizar el correspondiente análisis con el paquete estadístico para las ciencias sociales, SPSS, versión 24. Se aplicó la prueba Shapiro Wilk, para determinar si los datos presentaban una distribución normal: Todos los datos 
presentan normalidad en su distribución (Impulsividad, SP, BAS, ESP, REL, EE, BAP, RA, EC, RP, EDA y NEG), a excepción de la Estrategia de afrontamiento: autonomía. En la tabla 1 se presentan los puntajes de las participantes en las variables de impulsividad y estrategias de afrontamiento:

Tabla 2.

Puntaje de las participantes en impulsividad y estrategias de afrontamiento.

\begin{tabular}{lrr}
\hline & \multicolumn{2}{r}{$\begin{array}{r}\text { Desviación } \\
\text { estándar }\end{array}$} \\
\hline IMPULSIVIDAD & 44,07 & 13,755 \\
SP & 38,36 & 8,692 \\
BAS & 24,52 & 7,386 \\
ESP & 24 & 8,082 \\
REL & 29,95 & 9,37 \\
EE & 26,79 & 8,286 \\
BAP & 18,6 & 7,629 \\
RA & 12,21 & 5,201 \\
EC & 19,6 & 5,37 \\
RP & 21,55 & 6,094 \\
EDA & 11,02 & 3,174 \\
NEG & 9,12 & 3,263 \\
AUT & $\mathbf{3 , 6}$ & $\mathbf{1 , 7 8 1}$ \\
\hline
\end{tabular}

En los análisis estadísticos de las correlaciones de Pearson, para relacionar con Impulsividad, los datos de los Estilos de Afrontamiento con distribución normal (Impulsividad vs. SP, BAS, ESP, REL, EE, BAP, RA, EC, RP, EDA y NEG), los factores: ESP (Espera), RA (Reacción 
Agresiva) y EDA (Expresión de Dificultad de Afrontamiento), presentaron diferencias significativas $(<0.05)$, en relación con la impulsividad, que se describen en la tabla 2.

Tabla 3.

Correlación de Pearson entre impulsividad y estrategias de afrontamiento

\begin{tabular}{|c|c|c|c|c|c|c|c|c|c|c|c|c|}
\hline & & SP & BAS & ESP & REL & $\overline{\mathrm{EE}}$ & BAP & RA & EC & $\mathrm{RP}$ & EDA & NEG \\
\hline IMPULSIVIDAD & $\begin{array}{l}\text { Correlación de } \\
\text { Pearson }\end{array}$ & 0,145 & 0,043 &, $329 *$ & 0,043 & 0,292 & 0,123 &, $351 *$ & 0,173 & 0,059 &, $474 * *$ & 0,196 \\
\hline & Sig. (bilateral) & 0,36 & 0,788 & 0,033 & 0,786 & 0,061 & 0,437 & 0,023 & 0,274 & 0,712 & 0,002 & 0,213 \\
\hline
\end{tabular}

Las mujeres de la muestra evidencian: a mayor impulsividad, mayor espera, a mayor impulsividad mayor reacción agresiva y a mayor impulsividad mayor expresión de dificultad de afrontamiento.

De la prueba de Correlación no paramétrica de Rho Spearman, para relacionar impulsividad con los datos de los estilos de afrontamiento con distribución NO normal, (impulsividad vs. autonomía), no se encontraron diferencias significativas. En la comparación de medias de los datos distribuidos normalmente, se corrió una prueba T-Student, para contrastar convivencia vs. impulsividad y convivencia vs. estilos de afrontamiento, no se encontraron diferencias significativas.

Tabla 4.

Comparación de medias no paramétrica en factor autonomía

AUT

U de Mann-Whitney 146

Z $-1,994$


Sig. asintótica

(bilateral)

0,046

En la comparación de medias, de los datos NO distribuidos normalmente, se corrió una prueba Mann-Whitney, para contrastar convivencia vs. impulsividad y convivencia vs. estilos de afrontamiento, se encontró una diferencia significativa $(<0.05)$ en la estrategia de afrontamiento autonomía, contrastada con la variable de agrupación: toma de decisión (convivencia con agresor/ no convivencia con agresor). Tabla 3 y figura 1.

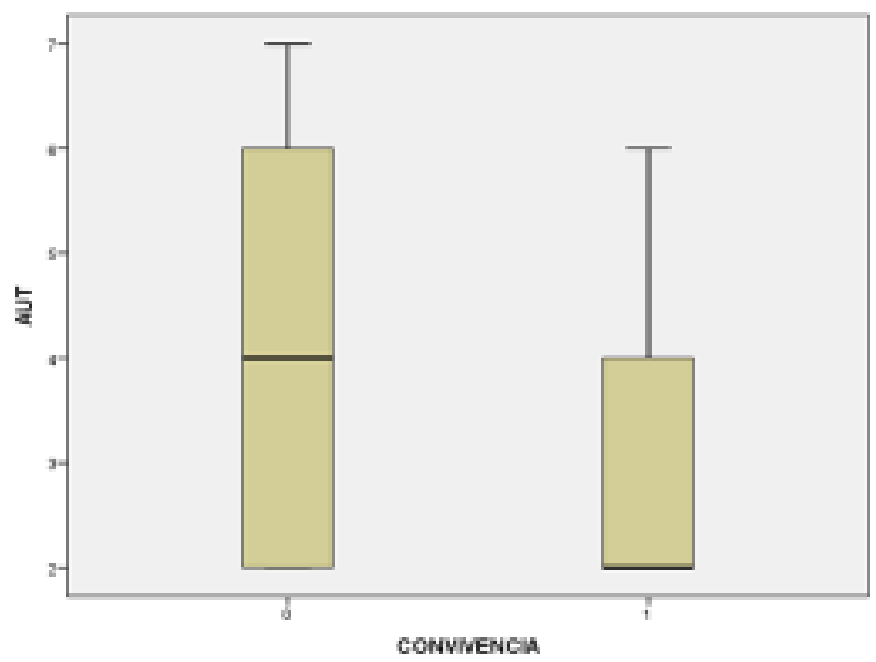

Figura 1. Diagrama de caja y bigotes para ambos grupos en el factor autonomía

Las mujeres de la muestra que no conviven con el agresor, presentan mayor autonomía como estrategia de afrontamiento.

\section{Discusión}

Al revisar los datos sociodemográficos de las mujeres del presente estudio, se registran edades entre los 19 y 63 años, los grupos más representativos están entre los 28 y 38 años, que concuerda con el estudio de la Secretaría Distrital de Integración Social (2015), donde las 
mujeres víctimas de violencia están principalmente entre los 27 y 59 años. Así mismo, se encontró que el $55 \%$ de las víctimas pertenecían al estrato 2, conforme a los resultados de la misma institución, donde se observó que la proporción más alta de víctimas reportadas ante las Comisarías de Familia, pertenecen especialmente al estrato 2 y por tanto, sugiere la mayor utilización por parte del estrato 2 de los servicios de las Comisarías.

Otro resultado reportado en el estudio, se refiere a las orientaciones recibidas por las víctimas por parte de la Comisaria de Familia: el $48 \%$ de las mujeres consideraron que fueron útiles y el 36\% claras; de igual forma, manifestaron que la medida de protección que se le otorgó, fue oportuna en un $52 \%$ y en un $31 \%$ efectiva, lo cual refleja, cómo la búsqueda de apoyo institucional es una estrategia de afrontamiento que cada vez está siendo más eficaz para que las mujeres víctimas de violencia de pareja tengan mayor resistencia al estrés, sean capaces de hacer frente a las experiencias maltratadoras y tomen mejores decisiones (Echeburúa et al., 2002).

Adicional a los hallazgos mencionados, se encontró relación entre los estilos de afrontamiento: espera, reacción agresiva y expresión de dificultad de afrontamiento, con impulsividad. Mestre et al. (2012); Lillo (2006) y Perona y Galán (2001), coinciden en que las estrategias de afrontamiento deben estar dirigidas y valoradas hacia la regulación de ciertos aspectos individuales, mantenimiento del equilibrio y potenciamiento del bienestar psicológico; por tanto, no se espera que una estrategia de afrontamiento exitosa esté relacionada con la impulsividad. De tal manera, los tres estilos de afrontamiento mencionados, relacionados con la impulsividad, pueden resultar en una ejecución menos eficaz. 
Contrario a lo esperado se evidenció que a mayor impulsividad hubo mayor espera como estrategia de afrontamiento, lo cual puede asociarse a los ciclos de violencia con períodos de tolerancia y descontrol. Labrador, et al. (2004) describen en este ciclo, la fase de acumulación de tensión caracterizada por agresiones, cambios en el estado de ánimo y como luego viene la calma, se evidencia que la mujer tiende a comportarse de forma sumisa, a ignorar y/o minimizar lo sucedido. Esta tolerancia, mantiene la violencia y hay un momento en que es tal el nivel de agotamiento, que conlleva a impulsividad y agresión por parte de ambos miembros de la pareja.

De la Rubia y López (2011) y De la Rubia, López, Díaz y Cienfuegos (2011), encontraron que las estrategias de afrontamiento pasivo como la evitación, desencadenan en mayor violencia y mayor permanencia en la relación; sin embargo, los resultados obtenidos en la presente investigación no evidenciaron relación entre el estilo de afrontamiento: evitación y los grupos de referencia. Por su parte, Zinc, Jacobson, Pabst, Regan y Fisher (2006) y Shannon, Logan, Cole y Medley (2006), indicaron en sus estudios, que la continuidad de relaciones de pareja violentas, se centraron en la búsqueda de ayuda profesional y búsqueda de apoyo social, en contraposición a los presentes hallazgos, debido a que no se encontraron diferencias significativas en ninguna de las variables mencionadas.

De igual manera los resultados del presente estudio, evidenciaron que el estilo de afrontamiento autonomía, se relacionó con la toma de decisión de continuar o no con la relación de pareja. En concordancia con estos hallazgos, Murray y Ali (2016), Frances (2005), Engin (2006) y Martínez, Piqueras e Inglés (2011), encontraron en sus respectivos trabajos, que los estilos de afrontamiento positivos y dirigidos hacia la toma de acciones por parte de las víctimas, tiene relación sobre toma de decisiones más exitosas y en la gestión de estados emocionales; de 
manera que las víctimas, resultan favorecidas con hacer frente a la acción y dar solución al problema.

En la presente investigación, los hallazgos indicaron que no se encontró relación entre la impulsividad y la toma de decisión (permanecer/abandonar la relación); en contraposición con los resultados obtenidos, los estudios previos en el tema que relacionaron impulsividad con toma de decisiones, encontraron que los individuos que exhibían alta impulsividad, presentaban déficit en la toma de decisiones, principalmente, en establecer asociaciones de recompensa y castigo, para tomar decisiones apropiadas (Aron, 2007; Ochoa, 2013 y Franken, Strien, Nijs y Muris, 2008).

Averdijk et al. (2016), plantean en su estudio que, haber sido víctima de violencia de pareja, tiene efectos sobre la valoración de los eventos y la toma de decisiones; de tal manera, que las víctimas perciben mayor los beneficios de la perpetración de violencia, que sus costos. Por tanto, se esperaría que las mujeres víctimas de violencia de pareja, tomen la decisión de continuar con la relación, contrario a los hallazgos de Copp, Giordano, Longmore y Manning (2015) y Rhatigan, Street y Axsom (2006), en las que la permanencia en la relación, es igual en mujeres violentadas y no violentadas en una relación.

Para la Secretaría Distrital de Integración Social (2015), se considera que existen diferencias en la configuración de la violencia en las parejas que conviven, las que no conviven y las que se encuentran en trance de separación. En el desarrollo del presente estudio, se encontró que es preciso diferenciar también, aquellas parejas que conviven, incluso comparten la misma cama pero ya no son pareja sentimental y no proyectan la separación.

Finalmente, como propuesta para futuros estudios, la presente investigación plantea la imperiosa necesidad de continuar investigando la violencia de pareja y de repensar no sólo la 
sociedad patriarcal, sino la distinción privado-público, el reforzamiento cultural, los conflictos humanos que alcanzan implicaciones jurídicas y propender por la defensa de los Derechos Humanos, la salud mental y el impacto de la violencia en la sociedad (Acevedo y Quiroga, 2015). Teniendo en cuenta que los factores socioculturales tuvieron un mayor impacto en la aceptación de la condición de violencia, se da una luz hacia los programas destinados a potenciar a la mujer y las estrategias de prevención competentes, clave para la reducción de la violencia de pareja (Mugoya, Witte y Ernst, 2015). Más aún, se sugiere desarrollar programas educativos que modifiquen patrones y valores sociales y culturales que logren la igualdad de género, acaben con la discriminación y con la violación de derechos humanos de la mujer (Rivas, 2015).

Adicional a lo anterior, se recomienda contar con un mayor universo muestral, con el fin de posibilitar la realización de más amplias generalizaciones, respecto a los resultados encontrados. De igual forma, sería provechoso replicar la investigación en otras localidades de Bogotá e incluso en otras regiones del país. Se propone también, la toma de una muestra aleatoria de mujeres víctimas de violencia de pareja (sin determinar convivencia o no), para identificar la relación entre historia de violencia y abandono de la pareja. Será fundamental seguir profundizando en esta problemática tan compleja como es la violencia de pareja, campo en donde la conducta tiene relación y consecuencia legales y la psicología jurídica puede seguir aportando amplia y positivamente.

\section{Conclusiones}

Los resultados de la presente investigación muestran la existencia de una correlación positiva entre las mujeres que no conviven con el agresor y las que presentan mayor autonomía como estilo de afrontamiento, así como entre impulsividad y reacción agresiva, e impulsividad con dificultad de afrontamiento, lo cual explica que se mantenga la problemática. Sin embargo, 
contrario a lo esperado se encontró que a mayor impulsividad hubo mayor espera, lo cual puede asociarse a los ciclos de violencia con períodos de tolerancia y descontrol.

Así mismo se evidenció en las mujeres del estudio que no existen correlaciones ni positivas ni negativas entre: impulsividad y convivencia con el agresor; entre impulsividad confrontada con los estilos de afrontamiento: solución de problemas, búsqueda de apoyo social, religión, evitación emocional, búsqueda de apoyo profesional, evitación cognitiva, reevaluación positiva, negación, autonomía. De igual manera se encontró que no existen correlaciones ni positivas ni negativas entre: convivencia y estilos de afrontamiento: solución de problemas, búsqueda de apoyo social, espera, religión, evitación emocional, búsqueda de apoyo profesional, reacción agresiva, evitación cognitiva, reevaluación positiva, expresión de dificultad de afrontamiento, negación.

\section{Referencias}

Acevedo, O. y Quiroga, L. (2015). Lineamientos de investigación y trabajos de grado. Manual básico. Maestría en Psicología Jurídica, Bogotá: Universidad Santo Tomas.

Aron, A. (2007). The neural basis of inhibition in cognitive control. The neuroscientist a review journal bringing neurobiology neurology and psychiatry, 13 (3), 214-228.

Averdijk, M., Van Gelder, J., Eisner, M. \& Ribeaud, D. (2016), Violence Begets Violence ... But How? A Decision-Making Perspective On The Victim-Offender Overlap. Criminology, $54(2), 282-306$.

Bell, K., \& Naugle, A. (2008). Intimate partner violence theoretical considerations: Moving towards a contextual framework. Clinical Psychology Review, 28, 1096-1107. Recuperado el 30 de junio de 2016 de: 


\section{http://www.academia.edu/7163377/Intimate_partner_violence_theoretical_considerations_Movi}

$\underline{\text { ng_towards_a_contextual_framework }}$

Breiding M., Basile K., Smith S., Black M. \& Mahendra R. (2015). Intimate Partner Violence Surveillance: Uniform Definitions and Recommended Data Elements. Atlanta (GA): National Center for Injury Prevention and Control, Centers for Disease Control and Prevention.

Campbell, J. (2002). Health consequences of intimate partner violence. Lancet. 359,13311336.

Cantón, J., Cortés, M., Justicia, M., Cantón, D. (2013) Violencia doméstica, divorcio y adaptación psicológica. España: Ediciones Pirámide

Casey, B., Somerville, L., Gotlib, I., Ayduk, O., Franklin, N., Askren, M. \& Shoda, Y. (2011). Behavioral and neural correlates of delay of gratification 40 years later. Proceedings of the National Academy of Sciences of the United States of America, 108 (36), 14998-15003.

Chahin, N., Cosi, S., Lorenzo-Seva, U., \& Vigil-Colet, A. (2005). Stability of the factor structure of Barrat's Impulsivity Scales for children across cultures: A comparison of Spain and Colombia. Psicothema, 22(4), 983-989.

Copp, J. E., Giordano, P. C., Longmore, M. A., \& Manning, W. D. (2015). Stay/Leave Decision-Making In Non-Violent And Violent Dating Relationships. Violence And Victims, 30(4), 581-599.

Corsi, J. (2004) Maltrato y abuso en el ámbito doméstico: fundamentos teóricos para el estudio de la violencia en las relaciones familiares. Buenos Aires: Paidós.

De la Rubia, J., López, F., Díaz, R. \& Cienfuegos, Y. (2011). Diferencias de Género en Afrontamiento y Violencia en la Pareja (Gender Differences In Coping And Violence In Couple Relationships). Revista CES Psicología, 4 (2), 29-46. 
Echeburúa, E., Corral, P. y Amor, P. (2002). Evaluación del daño psicológico en las víctimas de delitos violentos. Psicothema, 14, 139-146.

Engin, D. (2006). The Relationships Among Coping with Stress, Life Satisfaction, Decision-Making Styles and Decision Self-Esteem: An Investigation with Turkish University Student. Social Behavior and Personality: an international journal, 34 (9), 1161-1170.

Fernández-Abascal, E., y Palmero, F. (1999). Emociones y salud. Barcelona: Ariel Psicología.

Flórez, I., Herrera, E., Carpio, E., Veccino, M., Zambrano, D., Reyes, Y. \& Torres, S. (2011). Afrontamiento y adaptación en pacientes egresados de unidades de cuidado intensivo. Aquichan, 11 (1), 23-39.

Frances, L. (2005). Decision making as coping. Health Psychology, 24 (4), S23-S28.

Franken, H., Strien, J., Nijs, I. \& Muris, P. (2008). Impulsivity is associated with behavioral decision-making deficits. Psychiatry Research ,158 (2), 155 - 163.

Goodman, L., Dutton, M., Vankos, N. y Weinfurt, K. (2005). Womens resources and use of strategies as risk and protective factors for reabuse over time. Violence Against Women, 11, 311336.

Hernández, R., Fernández, C. y Baptista, P. (2010). Metodología de la Investigación. México, McGraw-Hill.

Haro, G., Castellano, M., Pérez, B., Rodríguez, E., Cervera, G. Y Valderrama, J. (2004). Revisión Histórica de la Impulsividad desde una Perspectiva Artística, Filosófica Y Psicopatológica. Salud Mental, 27 (5), 23-28.

Instituto Nacional de Medicina Legal y Ciencias Forenses (2013). Boletín de prensa Forensis, Bogotá. Recuperado noviembre 30 de 2014, de: 
http://www.medicinalegal.gov.co/documents/10180/145695/presentacion+forensis2014.pdf/3b0a a016-1a19-4322-9cf3-a9b9fd985978.

Instituto Nacional de Medicina Legal y Ciencias Forenses (2014). Forensis 2014 Datos para la vida, Bogotá. Recuperado marzo 1 de 2016, de: http://www.medicinalegal.gov.co/.../Forensis...JULpdf.pdf/9085ad79-d2a9-4c0d-...

Instituto Nacional de Medicina Legal y Ciencias Forenses (2015). Comportamiento de la Violencia de Pareja. Colombia, 2015. Bogotá. Recuperado agosto 27 de 2016, de: http://www.medicinalegal.gov.co/documents/88730/3418907/5.+VIOLENCIA+DE+PAREJA.pd f/03de1fd1-092d-495f-99d3-1aacee2991c6

Johnson, M. (2005). The Differential Effects of Intimate Terrorism and Situational Couple Violence: Findings from the National Violence Against Women Survey. Journal of Family Issues, 26 (3), 322-349.

Kidd, C., Palmeri, H. \& Aslin, R. (2013). Rational snacking: Young children’s decisionmaking on the marshmallow task is moderated by beliefs about environmental reliability. Cognition, 126 (1), 109-114.

Labrador, F., Rincón, P., Luis, P. y Fernández-Velasco, R. (2004). Mujeres víctimas de la violencia doméstica. Pirámide, Madrid.

Lazarus y Folkman (1984/1986). Emoción y procesos cognitivos. Barcelona: MartínezRoca.

Lillo, P. (2006). Influencia de los estilos de humor sobre las estrategias de afrontamiento en Entre Ríos. Psicodebate: Psicología, Cultura y Sociedad, 7, 95-118.

Londoño, N., Henao, G., Puerta, I., Posada, S., Arango, D. y Aguirre-Acevedo, D., Universidad de San Buenaventura, Medellín (2006). Propiedades psicométricas y validación de 
la Escala de Estrategias de Coping Modificada (EEC-M) en una muestra colombiana. Universitas Psychologica, 5 (2), 327-349.

Loyola, N. (2011). Validez y confiabilidad de la escala de impulsividad de Barratt versión 11 (bis-11) en mujeres encarceladas Pontificia Universidad Católica del Perú, Lima (2011). Recuperado el 11 de junio de 2016, de:

\section{file:///C:/Users/e_pal_000/Downloads/LOYOLA_ALVAREZ_NELLY_VALIDEZ\%20(2)\%20(}

4).pdf.

Martínez, A., Piqueras, J. E Inglés, C. (2011). Relaciones entre Inteligencia Emocional y Estrategias de Afrontamiento ante el Estrés. Revista electrónica de motivación y emoción, 14(37): 1-24, 92.

Martínez, A., Piqueras, J. y Ramos, V. (2010). Inteligencia emocional en la salud física y mental. Electronic Journal of Research in Educational Psychology. 21, 8(2), 861-890.

Martínez, J., Sánchez, J., Bechara, A. y Román, F. (2006). Mecanismos cerebrales de la toma de decisiones. RevistaNeurología, 42(7), 411-418.

Mestre, V., Samper, P., Tur-Porcar, A. M., Richaud de Minzi, M. C. y Mesurado, B. (2012). Emociones, estilos de afrontamiento y agresividad en la adolescencia. Universitas Psychologica, 11(4), 1263-1275.

Ministerio de Justicia y del Derecho. (2012). Justicia y Género, II Lineamientos técnicos en Violencias Basadas en Género para las Comisarías de Familia, Bogotá, D.C.

Morales, F. (2008). Evaluación del afrontamiento infantil: estudio inicial de las propiedades de un instrumento. Disertación doctoral no publicada. Universidad de Málaga, Málaga, España. Recuperado el 16 de junio de 2016, de http://riuma.uma.es/xmlui/handle/10630/2675 
Mugoya, G., Witte, T. \& Ernst, K. (2015). Sociocultural and Victimization Factors That Impact Attitudes Toward Intimate Partner Violence Among Kenyan Women. J Interpers Violence. 30 (16), 2851-2871.

Murray, P. \& Ali, F. (2016). Agency and coping strategies for ethnic and gendered minorities at work. The International Journal of Human Resource Management, 1-25. doi:http://dx.doi.org/10.1080/09585192.2016.1166787.

Ochoa, A. (2013). Estudio sobre toma de decisiones, el funcionamiento ejecutivo y la impulsividad en el juego patológico. Tesis Doctoral no Publicada. Universitat de Barcelona, Barcelona, España. Recuperado el 12 de junio de 2016, de http://diposit.ub.edu/dspace/bitstream/2445/36573/2/COA_TESIS.pdf.

Ojeda, A., Cuenca, J. Y Espinosa, D. (2008). Comunicación y afrontamiento como estrategias individuales que buscan facilitar la adaptación social en población migrante. Migración y Desarrollo, 11,79-95.

Organización Mundial de la Salud (2002) Informe mundial sobre la violencia y la salud Sinopsis, Ginebra. Recuperado agosto 27 de 2016, de:

http://www.who.int/violence_injury_prevention/violence/world_report/es/summary_es.pdf

Orozco-Cabal, L. y Herin, D. (2008). Neurobiología de la impulsividad y los trastornos de la conducta alimentaria. Revista Colombiana de Psiquiatría, 37(2), 207-219.

Perona, A. y Galán, S. (2001). Algunas aportaciones críticas en torno a la búsqueda de un marco teórico del afrontamiento en la psicosis. Psicothema, 13(4), 563-570.

Pontificia Universidad Javeriana (2007). Ley No1090 "Por la cual se reglamenta el ejercicio de la profesión de Psicología, se dicta el código deontológico y bioético y otras disposiciones". Editorial Pontificia Universidad Javeriana, Bogotá. 
Portilla, M., Bascarán, M., Sáiz, P., Bousoño, M., Bobes, J. (2005). Evaluación de la Impulsividad. Madrid.: Ars Médica,

Profamilia. (2010). Encuesta Nacional de Demografía y Salud. ENDS, Ministerio de Salud y la Protección Social, Bogotá, D.C.

Pueyo, A., S. López, S. y Álvarez, E. (2008) Valoración del riesgo de violencia contra la pareja por medio de la Sara. Grupo de Estudios Avanzados en Violencia. Facultad de PsicologíaUniversidad de Barcelona. Otras aportaciones. Papeles del Psicólogo, 2008. Vol. 29(1), pp. 107122. Recuperado el 30 de junio de 2016 de: http://www.papelesdelpsicologo.es/pdf/1543.pdf

Rhatigan, D., Street, A. \& Axsom, D. (2006). A critical review of theories to explain violent relationship termination: Implications for research and intervention. Clinical Psychology Review, 26(3), 321-345.

Reynolds, B. \& Schiffbauer, R. (2005). Delay of Gratification and Delay Discounting: A Unifying Feedback Model of Delay-Related Impulsive Behavior. The Psychological Record, 55: $439-460$

Rivas, M. (2015). La violencia contra la mujer: un grave flagelo universal. Victimología en América Latina Enfoque psicojurídico. Ediciones de la U, Bogotá.

Rolling, E. y Brosi, M. (2010). A multi-leveled and integrated approach to assessment and intervention of intimate partner violence. Journal of Family Violence, 25, 229-236.

Sánchez, M. (2015). La impulsividad y su influencia en el riesgo de violencia contra la pareja en las personas que acuden a la unidad judicial de violencia contra la mujer Ambato. Universidad Técnica de Ambato, Ecuador. Recuperado el 30 de junio de 2016 de: file://C:/Users/e_pal_000/Desktop/TESIS\%20MAYO\%202016/ARTICULOS/Sánchez\%20Cast ro,\%20María\%20José-impulsividad.pdf 
Sánchez, M., Encisco, M., Arenas, M., Vergara, R. Lanz, A., Ministerio de Justicia y del Derecho. (2012). Justicia y Género, I Marco Normativo en Torno a la Violencia Basada en Género. Bogotá, D.C.

Sánchez, P., Giraldo, J. \& Quiroz, M. (2013). Impulsividad: una visión desde la neurociencia del comportamiento y la psicología del desarrollo. Avances en Psicología Latinoamericana, 31(1), 241-251.

Secretaría Distrital de Integración Social (SDIS,2015). Las comisarías de familia de Bogotá y la atención de la violencia intrafamiliar. Caracterización de la violencia intrafamiliar a partir de la información contenida en el sistema de registro SIRBE. Bogotá, 2015.

Secretaría Distrital de Integración Social (SDIS,2015). Fortaleciendo habilidades para hacer frente y prevenir las violencias. Manual para la formación de servidores, servidoras, líderes, lideresas y organizaciones de base, como multiplicadoras y multiplicadores en prevención, orientación y atención de las violencias al interior de las familias. Bogotá, 2015.

Shannon, L., Logan, T., Cole, J. \& Medley, K. (2006). Help-Seeking and Coping Strategies for Intimate Partner Violence in Rural and Urban Women. Violence and Victims, 21 (2), 167181.

Squillace, M., Picón, J. \& Schmidt, V. (2011). El concepto de impulsividad y su ubicación en las teorías psicobiológicas de la personalidad. Revista Neuropsicología Latinoamericana.3 (1): 8-18.

Smith, G., Fischer, S., Cyders, M., Annus, A., Spillane, N. \& McCarthy, D. (2007). On the validity and utility of discriminating among impulsivity-like traits. Assessment, 14(2), 155-170.

Stanton, A. L. \& Dunkel-Schetter, C. (2013). Infertility: Perspectives from stress and coping research. Springer Science \& Business Media. 
Swann, A., Bjork, J., Moeller, F. \& Dougherty, D. (2002). Two models of impulsivity: relationship to personality traits and psychopathology. Biol Psychiatry, 51 (12), 988-994.

Taggart, S. (2009). Decision making and Domestic Violence: An Advanced Training for TDM Facilitators and Child Protection Supervisors Trainer's Guide. Family Violence Prevention Fund Team.

Vásquez, B. (2007). Manual de Psicología Forense. Editorial Síntesis, Madrid.

Vázquez. C., Crespo. M. y Ring. J. (2003). Estrategias de afrontamiento. Medición clínica en psiquiatría y psicología. Cáp. 31, pp. 425-35

Veloza, M., Moreno, M., Crespo, O y Gutiérrez E. (2007). Proceso de afrontamiento y adaptación. Adaptación y cuidado en el ser humano. Una visión de enfermería. Bogotá: El Manual Moderno: pp.13-25.

Winstanley, C., Eagle, D. \& Robbins, T. (2006). Behavioral models of impulsivity in relation to ADHD: Translation between clinical and preclinical studies. Clinical Psychologt Review, 26(4), 379-395.

Zambrano, J., Valle, M., Macías, M. \& Madariaga, C. (2013). Estrategias de afrontamiento individual y familiar frente a situaciones de estrés psicológico. Psicología desde el Caribe, 30, $123-145$.

Zinc, T., Jacobson, C., Pabst, S., Regan, S. \& Fisher, B. (2006).A Lifetime of Intimate Partner Violence Coping Strategies of Older Women. J Interpers Violence, 21 (5), 634-651. 


\section{ANEXOS \\ N. UNIVERSIDA SANTT TOMAS \\ FACULTAD DE PSICOLOGÍA \\ MAESTRÍA EN PSICOLOGÍA JURÍDICA \\ CONSENTIMIENTO INFORMADO}

Yo (Nombres completo)

mayor de edad e identificada con C.C.

manifiesto

que acepto participar en la investigación de tesis de grado titulada "Relación entre impulsividad, afrontamiento y toma de decisiones de continuar o no la relación con el agresor en mujeres que denunciaron violencia pareja" realizada por la Psicóloga Esperanza Palacios Cortes.

El objetivo me ha sido mencionado claramente, he sido informada sobre el propósito y manejo de la información recolectada.

Mi participación es voluntaria y puedo tomar la decisión de retirarme tranquilamente en cualquier momento si así lo deseo, comprendo que mi nombre no será divulgado y se conservará en el anonimato.

Autorizo la aplicación de pruebas psicológicas y entiendo que la información derivada de esta investigación será publicada en un informe científico y que puedo tener acceso a los resultados personales.

Firma

Fecha 


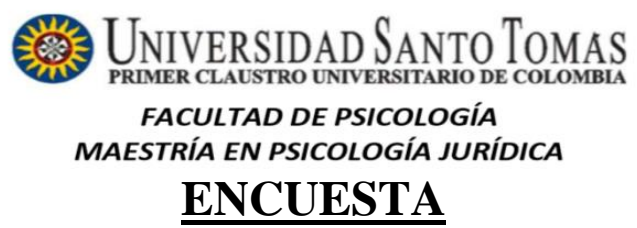

1. ¿Conocía Ud. sus derechos fundamentales como mujer antes de acudir a la Comisaria de Familia?

- $\mathrm{SI}$

- NO

2. Las orientaciones que recibió por parte de la Comisaria de Familia fueron:

a) útiles

b) claras

c) oportunas

d) confusas

e) inadecuadas

f) insuficientes

3. Considera que la medida de protección que se le dio por parte de la Comisaria de Familia fue:
a) oportuna
b) efectiva
c) accesible
d) desproporcionada
e) Inútil
f) ineficiente

4. ¿Las orientaciones que recibió por parte de la Comisaria de Familia influyeron en la toma de decisión de separarse o no de su pareja?

- SI

- NO

5. ¿Después de la medida de protección Ud. continúa viviendo con su pareja?

- SI

- NO 


\section{Escala de Impulsividad de Barrat}

(Barratt Impulsiveness Scale, BIS-11)

Instrucciones Las Personas son diferentes en cuanto a la forma en que se comportan y piensan en distintas situaciones. Esta es una escala para medir algunas de las formas en que usted actúa y piensa. No se detenga demasiado tiempo en las oraciones. Responda rápida y honestamente marcando una $X$ en la opción que más le represente. (Entrevistador: lea cada oración al entrevistado y marque la respuesta. Si no entiende la pregunta o si es más atingente plantéele la forma entre paréntesis.)

\begin{tabular}{|c|c|c|c|c|}
\hline & $\begin{array}{l}\text { Raramente } \\
\text { o Nunca } \\
(0)\end{array}$ & $\begin{array}{l}\text { Ocasionalmente } \\
\text { (1) }\end{array}$ & $\begin{array}{l}\text { A menudo } \\
\text { (3) }\end{array}$ & $\begin{array}{c}\text { Siempre o } \\
\text { Casi Siempre } \\
(4)\end{array}$ \\
\hline $\begin{array}{l}1 \text { Planifico mis tareas con } \\
\text { cuidado }\end{array}$ & $\square$ & $\square$ & $\square$ & $\square$ \\
\hline 2 Hago las cosas sin pensarlas & $\square$ & $\square$ & $\square$ & $\square$ \\
\hline $\begin{array}{l}3 \text { Casi nunca me tomo las } \\
\text { cosas a pecho (no me perturbo } \\
\text { fácilmente) }\end{array}$ & $\square$ & $\square$ & $\square$ & $\square$ \\
\hline $\begin{array}{l}4 \text { Mis pensamientos pueden } \\
\text { tener gran velocidad (mis } \\
\text { pensamientos van muy rápido } \\
\text { en mi mente) }\end{array}$ & $\square$ & $\square$ & $\square$ & $\square$ \\
\hline $\begin{array}{l}5 \text { Planifico mis viajes } \\
\text { (actividades) con antelación }\end{array}$ & $\square$ & $\square$ & $\square$ & $\square$ \\
\hline $\begin{array}{l}6 \text { Soy una persona con } \\
\text { autocontrol }\end{array}$ & $\square$ & $\square$ & $\square$ & $\square$ \\
\hline $7 \mathrm{Me}$ concentro con facilidad & $\square$ & $\square$ & $\square$ & $\square$ \\
\hline 8 Ahorro con regularidad & $\square$ & $\square$ & $\square$ & $\square$ \\
\hline $\begin{array}{l}9 \text { Se me hace difícil estar quieto } \\
\text { por largos períodos de tiempo }\end{array}$ & $\square$ & $\square$ & $\square$ & $\square$ \\
\hline $\begin{array}{l}10 \text { Pienso las cosas } \\
\text { cuidadosamente }\end{array}$ & $\square$ & $\square$ & $\square$ & $\square$ \\
\hline $\begin{array}{l}11 \text { Planifico para tener un } \\
\text { trabajo fijo (me esfuerzo para } \\
\text { asegurarme de que tendré } \\
\text { dinero para pagar mis gastos) }\end{array}$ & $\square$ & $\square$ & $\square$ & $\square$ \\
\hline 12 Digo las cosas sin pensarlas & $\square$ & $\square$ & $\square$ & $\square$ \\
\hline $\begin{array}{l}13 \text { Me gusta pensar sobre } \\
\text { problemas complicados (me } \\
\text { gusta pensar sobre problemas } \\
\text { complejos) }\end{array}$ & $\square$ & $\square$ & $\square$ & $\square$ \\
\hline $\begin{array}{l}14 \text { Cambio de trabajo } \\
\text { frecuentemente (no me quedo } \\
\text { en el mismo trabajo por largos } \\
\text { períodos de tiempo) }\end{array}$ & $\square$ & $\square$ & $\square$ & $\square$ \\
\hline 15 Actúo impulsivamente & $\square$ & $\square$ & $\square$ & $\square$ \\
\hline
\end{tabular}




\begin{tabular}{|c|c|c|c|c|}
\hline $\begin{array}{l}16 \mathrm{Me} \text { aburro con facilidad } \\
\text { tratando de resolver problemas } \\
\text { en mi mente (me aburre pensar } \\
\text { en algo por demasiado tiempo) }\end{array}$ & $\square$ & $\square$ & $\square$ & $\square$ \\
\hline $\begin{array}{l}17 \text { Visito al médico y al dentista } \\
\text { con regularidad }\end{array}$ & $\square$ & $\square$ & $\square$ & $\square$ \\
\hline $\begin{array}{l}18 \text { Hago las cosas en el } \\
\text { momento que se me ocurren }\end{array}$ & $\square$ & $\square$ & $\square$ & $\square$ \\
\hline $\begin{array}{l}19 \text { Soy una persona que piensa } \\
\text { sin distraerse (puedo enfocar mi } \\
\text { mente en una sola cosa por } \\
\text { mucho tiempo) }\end{array}$ & $\square$ & $\square$ & $\square$ & $\square$ \\
\hline $\begin{array}{l}20 \text { Cambio de vivienda a } \\
\text { menudo (me mudo con } \\
\text { frecuencia o no me gusta vivir } \\
\text { en el mismo sitio por mucho } \\
\text { tiempo) }\end{array}$ & $\square$ & $\square$ & $\square$ & $\square$ \\
\hline $\begin{array}{l}21 \text { Compro cosas } \\
\text { impulsivamente }\end{array}$ & $\square$ & $\square$ & $\square$ & $\square$ \\
\hline 22 Termino lo que empiezo & $\square$ & $\square$ & $\square$ & $\square$ \\
\hline $\begin{array}{l}23 \text { Camino y me muevo con } \\
\text { rapidez }\end{array}$ & $\square$ & $\square$ & $\square$ & $\square$ \\
\hline $\begin{array}{l}24 \text { Resuelvo los problemas } \\
\text { experimentando (resuelvo los } \\
\text { problemas empleando una } \\
\text { posible solución y viendo si } \\
\text { funciona) }\end{array}$ & $\square$ & $\square$ & $\square$ & $\square$ \\
\hline $\begin{array}{l}25 \text { Gasto en efectivo o a } \\
\text { crédito más de lo que gano } \\
\text { (gasto más de lo que gano) }\end{array}$ & $\square$ & $\square$ & $\square$ & $\square$ \\
\hline 26 Hablo rápido & $\square$ & $\square$ & $\square$ & $\square$ \\
\hline $\begin{array}{l}27 \text { Tengo pensamientos } \\
\text { extraños cuando estoy } \\
\text { pensando (a veces tengo } \\
\text { pensamientos irrelevantes } \\
\text { cuando pienso) }\end{array}$ & $\square$ & $\square$ & $\square$ & $\square$ \\
\hline $\begin{array}{l}28 \mathrm{Me} \text { interesa más el presente } \\
\text { que el futuro }\end{array}$ & $\square$ & $\square$ & $\square$ & $\square$ \\
\hline $\begin{array}{l}29 \text { Me siento inquieto/a en } \\
\text { clases o charlas (me siento } \\
\text { inquieto/a si tengo que oír a } \\
\text { alguien hablar demasiado } \\
\text { tiempo) }\end{array}$ & $\square$ & $\square$ & $\square$ & $\square$ \\
\hline $\begin{array}{l}30 \text { Planifico el futuro (Me } \\
\text { interesa más el futuro que el } \\
\text { presente) }\end{array}$ & $\square$ & $\square$ & $\square$ & $\square$ \\
\hline
\end{tabular}




\section{ESCALA DE ESTRATEGIAS DE COPING- MODIFICADA (EEC-M)}

Londoño N H, Henao G C, Puerta I C, Posada S L, Arango D, Aguirre, D C

Grupo de Investigación Estudios Clínicos y Sociales en Psicología

Universidad de San Buenaventura, Medellín, Colombia

Instrucciones: A continuación se plantean diferentes formas que emplean las personas para afrontar los problemas o situaciones estresantes que se les presentan en la vida Las formas de afrontamiento aquí descritas no son ni buenas ni malas, ni mejores o peores Simplemente ciertas personas utilizan unas formas más que otras, dependiendo de la situación problema Trate de recordar las diferentes situaciones o problemas más estresantes vividos durante los últimos años, y responda señalando con una $X$ en la columna que le señala la flecha $(\downarrow)$, el número que mejor indique qué tan habitual ha sido esta forma de comportamiento ante las situaciones estresantes 1
2
3
4
5
Nunca
Casi nunca A veces
Frecuentemente
Casi siempre Siempre

\begin{tabular}{|c|c|c|c|c|c|c|c|c|c|c|c|c|c|c|}
\hline & & $\downarrow$ & 1 & 2 & 3 & 4 & 5 & 6 & 7 & 8 & 9 & 10 & 11 & 12 \\
\hline 1 & $\begin{array}{l}\text { Trato de comportarme } \\
\text { como si nada hubiera } \\
\text { pasado }\end{array}$ & 123456 & & & & & & & & & & & & \\
\hline 2 & $\begin{array}{l}\text { Me alejo del problema } \\
\text { temporalmente } \\
\text { (tomando unas } \\
\text { vacaciones, } \\
\text { descansando,etc) } \\
\end{array}$ & 123456 & & & & & & & & & & & & \\
\hline 3 & $\begin{array}{l}\text { Procuro no pensar en el } \\
\text { problema }\end{array}$ & 123456 & & & & & & & & & & & & \\
\hline 4 & $\begin{array}{l}\text { Descargo mi mal humor } \\
\text { con los demás }\end{array}$ & 123456 & & & & & & & & & & & & \\
\hline 5 & $\begin{array}{l}\text { Intento ver los aspectos } \\
\text { positivos del problema }\end{array}$ & 123456 & & & & & & & & & & & & \\
\hline 6 & $\begin{array}{l}\text { Le cuento a familiares o } \\
\text { amigos cómo me siento }\end{array}$ & 123456 & & & & & & & & & & & & \\
\hline 7 & $\begin{array}{l}\text { Procuro conocer mejor } \\
\text { el problema con ayuda } \\
\text { de un profesional }\end{array}$ & 123456 & & & & & & & & & & & & \\
\hline 8 & Asisto a la iglesia & 123456 & & & & & & & & & & & & \\
\hline 9 & $\begin{array}{l}\text { Espero que la solución } \\
\text { llegue sola }\end{array}$ & 123456 & & & & & & & & & & & & \\
\hline 10 & $\begin{array}{l}\text { Trato de solucionar el } \\
\text { problema siguiendo } \\
\text { unos pasos concretos } \\
\text { bien pensados }\end{array}$ & 123456 & & & & & & & & & & & & \\
\hline 11 & $\begin{array}{l}\text { Procuro guardar para mí } \\
\text { los sentimientos }\end{array}$ & 123456 & & & & & & & & & & & & \\
\hline 12 & $\begin{array}{l}\text { Me comporto de forma } \\
\text { hostil con los demás }\end{array}$ & 123456 & & & & & & & & & & & & \\
\hline 13 & $\begin{array}{l}\text { Intento sacar algo } \\
\text { positivo del problema }\end{array}$ & 123456 & & & & & & & & & & & & \\
\hline 14 & $\begin{array}{l}\text { Pido consejo u } \\
\text { orientación a algún } \\
\text { pariente o amigo para } \\
\text { poder afrontar mejor el } \\
\text { problema }\end{array}$ & 123456 & & & & & & & & & & & & \\
\hline
\end{tabular}




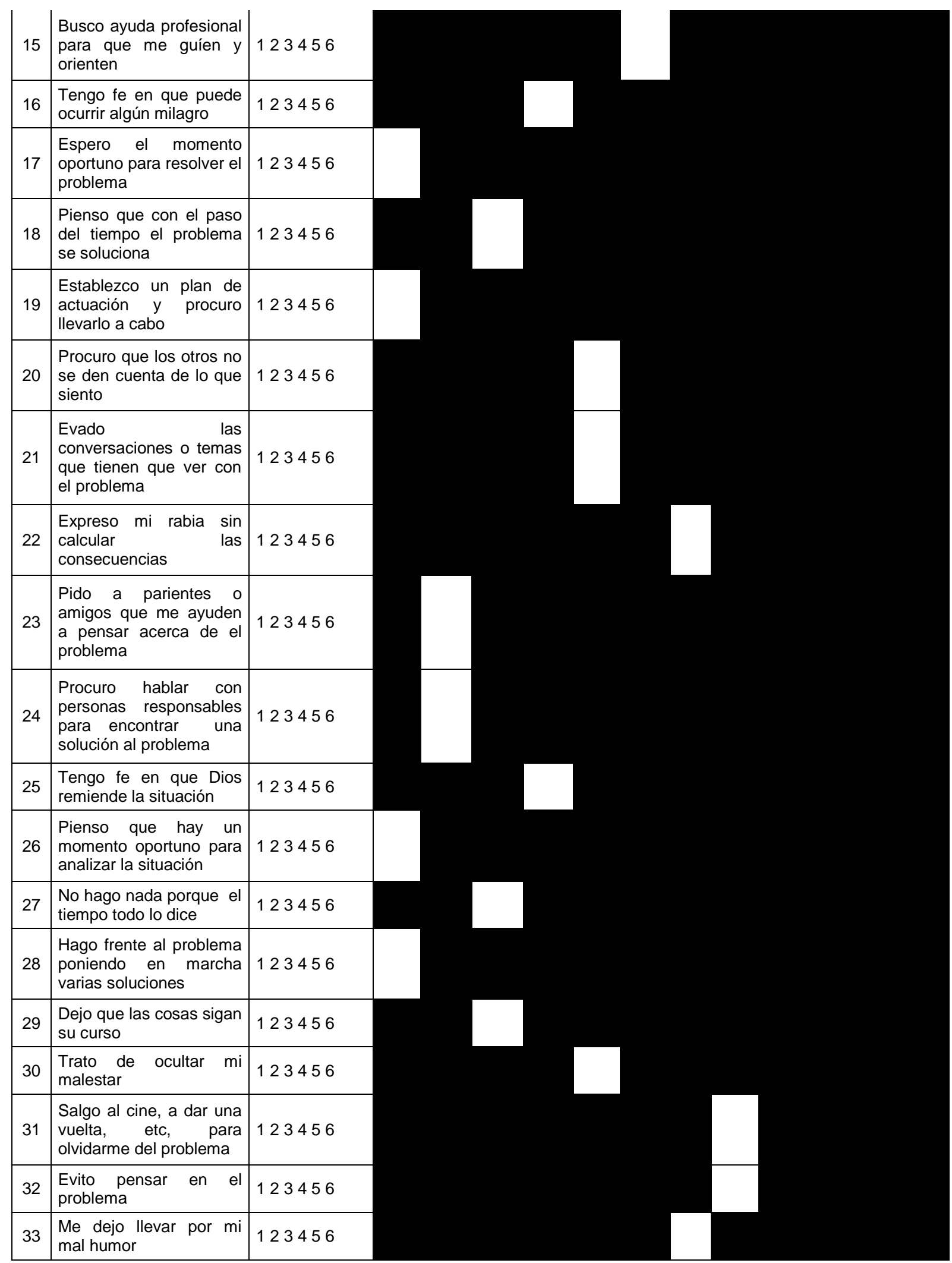




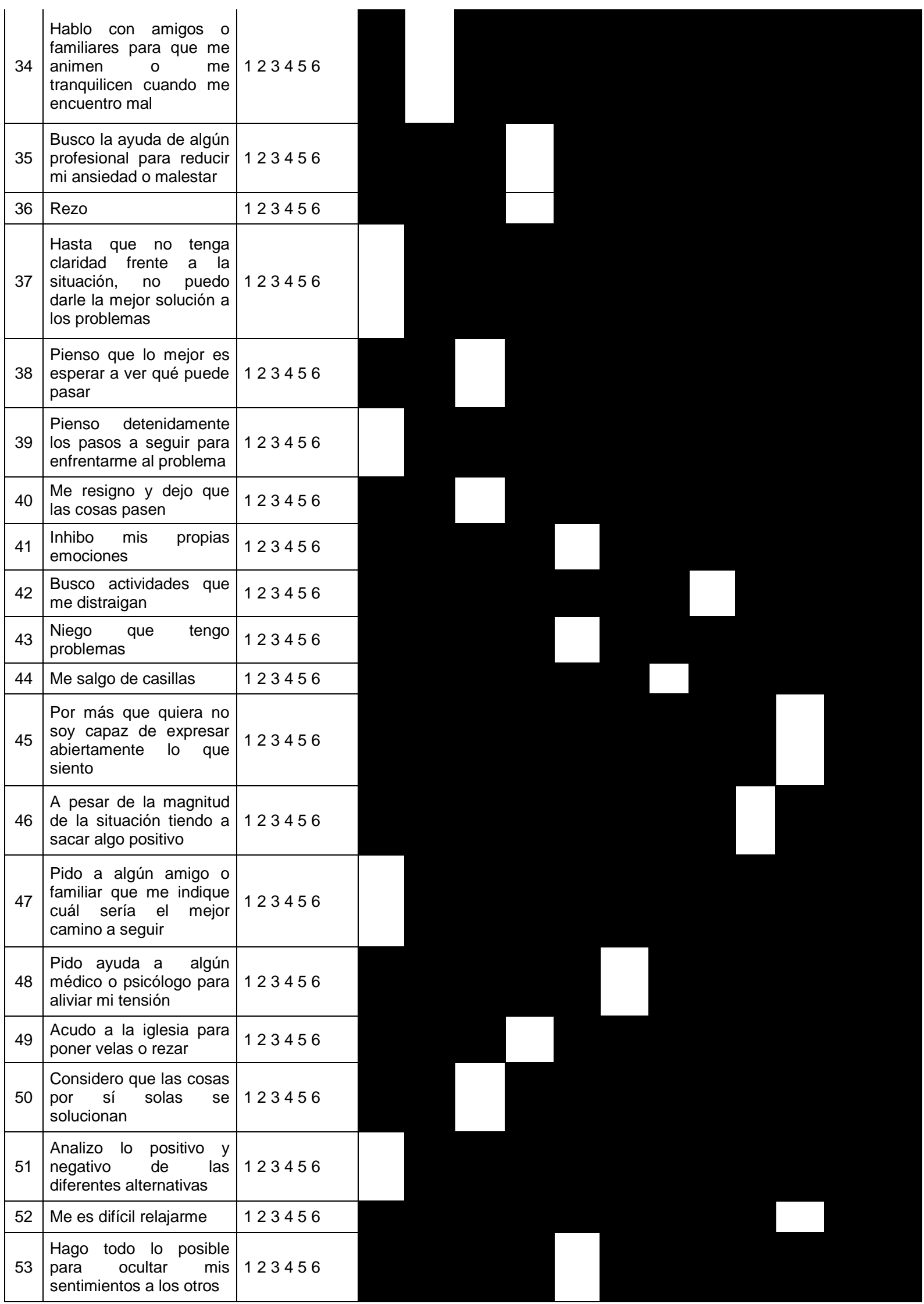




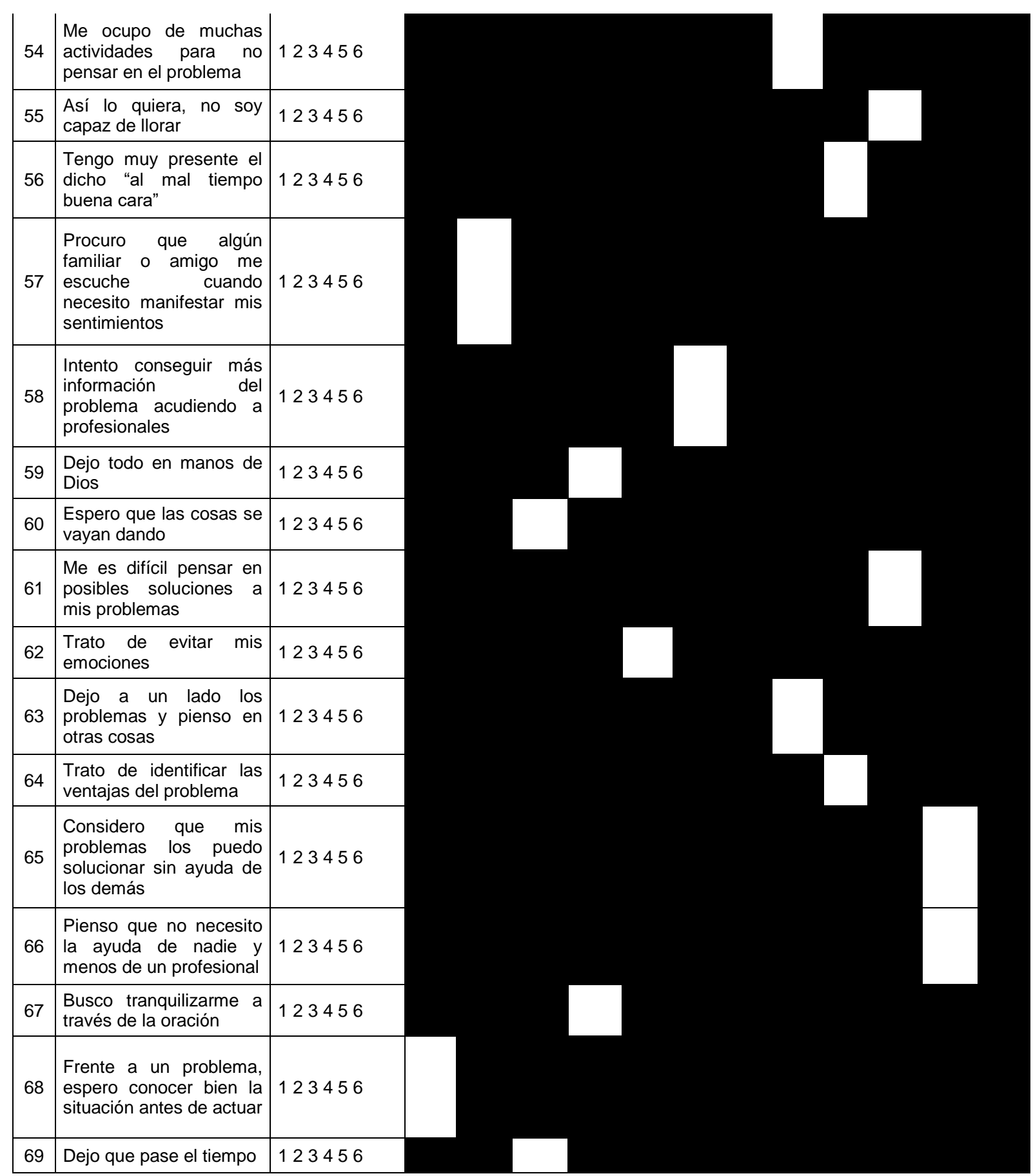

1 Univ Psychol Bogotá (Colombia), 5 (2) 327-349, mayo-agosto de 2006 


\section{Población}

\section{Datos sociodemográficos:}

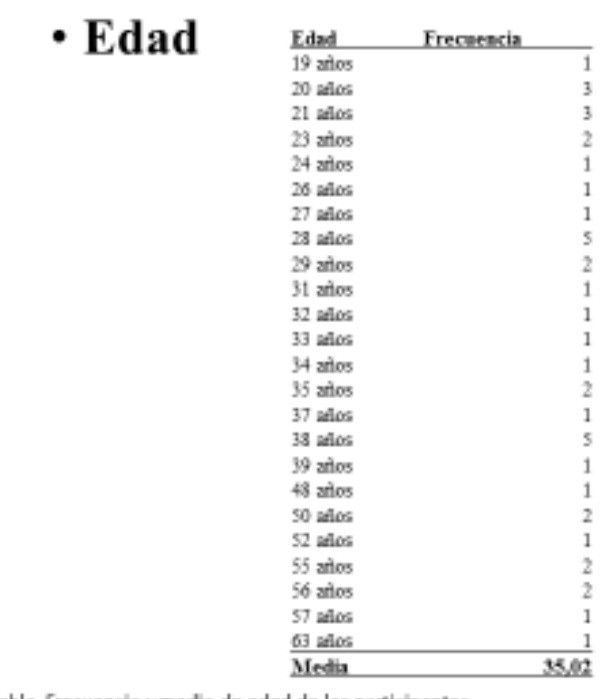

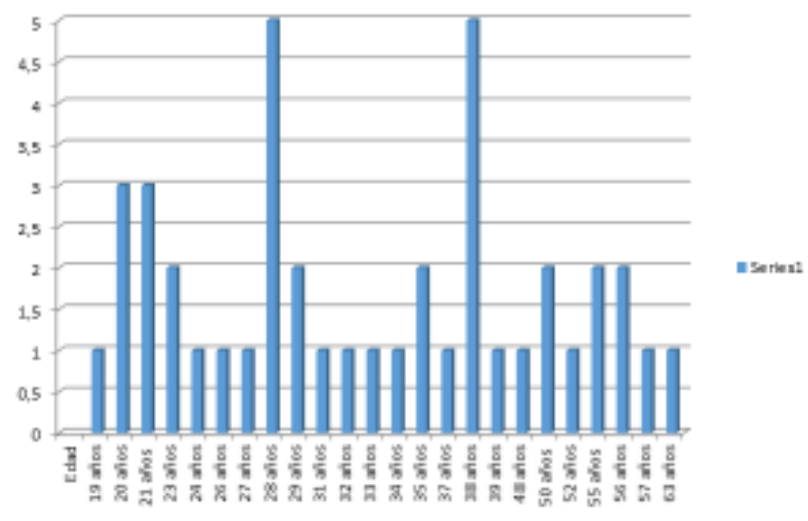

Figura. Columna gráfica de edades de las participantes

\section{Población}

\section{Datos sociodemográficos}

\section{- Nivel educativo}

\begin{tabular}{lr} 
Nivel Educativo & Frecuencia \\
\hline Primaria Incompleta & 1 \\
Primaria & 7 \\
Bachillerato Incompleto & 12 \\
Bachillerato & 10 \\
Técnico & 5 \\
Universitaria Incompleta & 4 \\
Universitaria & 2 \\
Especialización & 1 \\
\hline
\end{tabular}

Tabla. Nivel educativo de la muestra

\section{Gráfico Nivel Educativo}

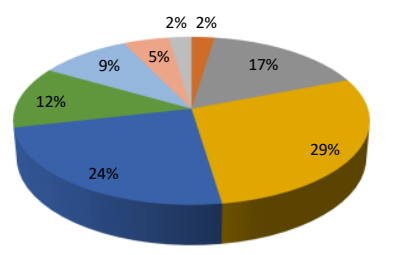

- Nivel Educativo

- Primaria Incompleta

$\because$ Primaria

- Bachillerato Incompleto

- Bachillerato

- Técnico

- Universitaria Incompleta

घ Universitaria

Especialización

Figura. Gráfico de tortas nivel educativo de la muestra 


\section{Población}

\section{Datos Sociodemográficos}

\section{- Estrato socioeconómico}

\begin{tabular}{lr} 
Estrato & Frecuencia \\
\hline Estrato 1 & 7 \\
Estrato 2 & 23 \\
Estrato 3 & 3 \\
Estrato 4 & 7 \\
Estrato 5 & 2 \\
\hline
\end{tabular}

Tabla. Estrato de la muestra

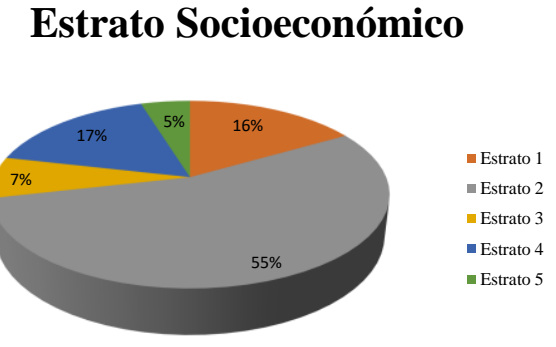

Figura. Gráfica de tortas del estrato de la muestra

\section{Resultados}

Encuesta General sobre la Medida de Protección

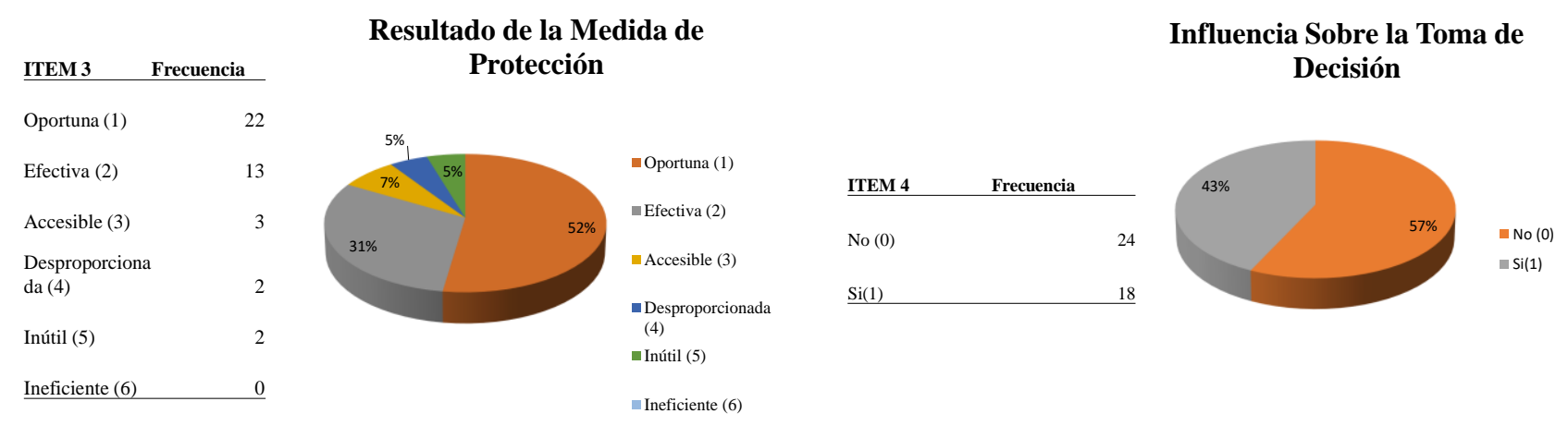




\section{Resultados}

Encuesta General sobre la Medida de Protección

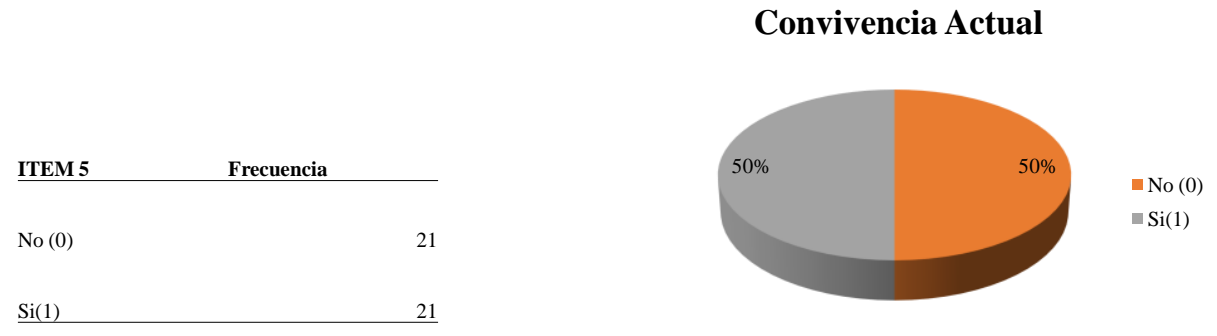

